\title{
A Complexity-Based Hierarchy for Multiprocessor Synchronization
}

\author{
[Extended Abstract]
}

\author{
Faith Ellen \\ University of Toronto \\ faith@cs.toronto.edu \\ Nir Shavit \\ MIT \\ shanir@csail.mit.edu
}

\author{
Rati Gelashvili \\ MIT \\ gelash@mit.edu \\ Leqi Zhu \\ University of Toronto \\ lezhu@cs.toronto.edu
}

\begin{abstract}
For many years, Herlihy's elegant computability based Consensus Hierarchy has been our best explanation of the relative power of various types of multiprocessor synchronization objects when used in deterministic algorithms. However, key to this hierarchy is treating these instructions as distinct objects, an approach that is far from the real-world, where multiprocessor programs apply synchronization instructions to collections of arbitrary memory locations. We were surprised to realize that, when considering instructions applied to memory locations, the computability based hierarchy collapses. This leaves open the question of how to better captures the power of various synchronization instructions.

In this paper, we provide an approach to answering this question. We present a hierarchy of synchronization instructions, classified by their space complexity in solving obstruction-free consensus. Our hierarchy provides a classification of combinations of known instructions that seems to fit with our intuition of how useful some are in practice, while questioning the effectiveness of others. We prove an essentially tight characterization of the power of buffered read and write instructions. Interestingly, we show a similar result for multi-location atomic assignments.
\end{abstract}

\section{INTRODUCTION}

Herlihy's Consensus Hierarchy [Her91] assigns a consensus number to each object, namely, the number of processes for which there is a wait-free binary consensus algorithm using only instances of this object and read-write registers. It is simple, elegant and, for many years, has been our best explanation of synchronization power.

Robustness says that, using combinations of objects with consensus numbers at most $k$, it is not possible to solve wait-free consensus for more than $k$ processes [Jay93]. The

Permission to make digital or hard copies of all or part of this work for personal or classroom use is granted without fee provided that copies are not made or distributed for profit or commercial advantage and that copies bear this notice and the full citation on the first page. Copyrights for components of this work owned by others than ACM must be honored. Abstracting with credit is permitted. To copy otherwise, or republish, to post on servers or to redistribute to lists, requires prior specific permission and/or a fee. Request permissions from permissions@ acm.org.

PODC'16, July 25-28, 2016, Chicago, IL, USA

(C) 2016 ACM. ISBN 978-1-4503-3964-3/16/07 . \$ $\$ 15.00$

DOI: http://dx.doi.org/10.1145/2933057.2933113 implication is that modern machines need to provide objects with infinite consensus number. Otherwise, they will not be universal, that is, they cannot be used to implement all objects or solve all tasks in a wait-free (or non-blocking) manner for any number of processes [Her91, Tau06, Ray12, HS12]. Although there are ingenious non-deterministic constructions that prove that Herlihy's Consensus Hierarchy is not robust [Sch97, LH00], it is known to be robust for deterministic one-shot objects [HR00] and deterministic readmodify-write and readable objects [Rup00]. It is unknown whether it is robust for general deterministic objects.

In adopting this explanation of computational power, we failed to notice an important fact: multiprocessors do not compute using synchronization objects. Rather, they apply synchronization instructions to locations in memory. With this point of view, Herlihy's Consensus Hierarchy no longer captures the phenomena we are trying to explain.

For example, consider two simple instructions:

- fetch-and-add(2), which returns the number stored in a memory location and increases its value by 2 , and

- test-and-set(), which returns the number stored in a memory location and sets it to 1 if it contained $0^{1}$.

Objects that support only one of these instructions as an operation have consensus number 2 and cannot be combined to solve wait-free consensus for 3 or more processes. However, a system that supports both instructions can solve wait-free binary consensus for any number of processes. The protocol uses a single memory location initialized to 0 . Processes with input 0 perform fetch-and-add(2), while processes with input 1 perform test-and-set(). If the value returned is odd, the process decides 1 . If the value 0 was returned from test-and-set(), the process also decides 1 . Otherwise, the process decides 0 .

Another example considers three instructions:

- $\operatorname{read}()$, which returns the number stored in a memory location,

- decrement(), which decrements the number stored in a memory location and returns nothing, and

\footnotetext{
${ }^{1}$ This definition of test-and-set is slightly stronger than the standard one, which simply always sets the location to 1 . However, they have the same consensus number and they behave identically if the values are always binary.
} 
- multiply $(x)$, which multiplies the number stored in a memory location by $x$ and returns nothing.

A similar situation arises: Objects that support read and one of decrement and multiply have consensus number 1 and cannot be combined to solve wait-free consensus for 2 or more processes. However, a system that supports all three instructions can solve wait-free binary consensus for any number of processes. The protocol uses a single memory location initialized to 1 . Processes with input 0 perform decrement(), while processes with input 1 perform multiply $(n)$. The second operation by each process is read(). If the value returned is positive, then the process decides 1 . If it is negative, then the process decides 0 .

For randomized computation, Herlihy's Consensus Hierarchy also collapses: randomized wait-free binary consensus among any number of processes can be solved using only read-write registers, which have consensus number 1. Fich, Herlihy, and Shavit [FHS98] proved that $\Omega(\sqrt{n})$ historyless objects, which support only trivial operations, such as read, and historyless operations, such as write, test-and-set, and swap, are necessary to solve this problem. They noted that, in contrast, only one fetch-and-increment or fetch-and-add object suffices for solving this problem. Yet, these objects and historyless objects are similarly classified in Herlihy's Consensus Hierarchy (i.e. they all have consensus number 1 or 2). They suggested that the number of instances of an object needed to solve randomized wait-free consensus among $n$ processes might be another way to classifying its power.

Motivated by these observations, we consider a classification of instruction sets based on the number of memory locations needed to solve obstruction-free $n$-valued consensus among $n \geq 2$ processes. Obstruction freedom is a simple and natural progress measure. Some state-of-the-art synchronization operations, for example hardware transactions [Int12], do not guarantee more than obstruction freedom. Obstruction freedom is also closely related to randomized computation. In fact, any (deterministic) obstruction free algorithm can be transformed into a randomized wait-free algorithm that uses the same number of memory locations (against an oblivious adversary) [GHHW13]. Obstructionfree algorithms can also be transformed into wait-free algorithms in the unknown-bound semi-synchronous model [FLMS05].

\subsection{Our Results}

For various instruction sets $\mathcal{I}$, we provide upper and lower bounds on $\mathcal{S P}(\mathcal{I}, n)$, the number of memory locations (supporting $\mathcal{I}$ ) needed to solve obstruction-free $n$-valued consensus among $n \geq 2$ processes (abbreviated as $n$-consensus). The results are summarized in Table 1 .

We begin, in Section 3, by considering the instructions

- multiply $(x)$, which multiplies the number stored in a memory location by $x$ and returns nothing, and

- $\operatorname{add}(x)$, which adds $x$ to the number stored in a memory location and returns nothing.

We show that one memory location supporting read() and one of these instructions can be used to solve $n$-consensus. The idea is to show that these instruction sets can implement $n$ counters in a single location. We can then use a racing counters algorithm.
Next, we consider max-registers [AAC09], which are memory locations supporting

- read-max (), which reads the number stored in a memory location, and

- write-max $(x)$, which stores the number $x$ in a memory location, provided it contains a value less than $x$, and returns nothing.

In Section 4, we prove that two max registers are necessary and sufficient for solving $n$-consensus.

In Section 5, we prove that a single memory location supporting $\{\operatorname{read}()$, write $(x)$, fetch-and-increment ()$\}$ cannot be used to solve $n$-consensus, for $n \geq 3$. We also present an algorithm for solving $n$-consensus using $O(\log n)$ such memory locations.

In Section 6, we introduce a family of instructions $\mathcal{B}_{\ell}$, for $\ell \geq 1$, and show how to solve $n$-consensus using $\left\lceil\frac{n}{\ell}\right\rceil$ memory locations supporting these instructions. Extending Zhu's $n-1$ lower bound [Zhu16], we also prove that $\left\lceil\frac{n-1}{\ell}\right\rceil$ such memory locations are necessary, which is tight except when $n-1$ is divisible by $\ell$.

Our main technical contribution is in Section 7, where we show that the preceding lower bound holds within a factor of two even in the presence of atomic multiple assignment. Multiple assignment can be implemented by simple transactions, so our result implies that such transactions cannot significantly reduce space complexity. The proof further extends the techniques of [Zhu16] via a nice combinatorial argument, which we hope will be of independent interest.

There are algorithms that solve $n$-consensus using $n$ registers [AH90, BRS15, Zhu15]. The best-known lower bound is $n-1$ registers [Zhu16]. A modification of the anonymous algorithm for $n$-consensus in [Zhu15] allows us to use only $n-1$ memory locations supporting $\{\operatorname{read}(), \operatorname{swap}(x)\}$. It is presented in Section 8. We also explain how to adapt the lower bound in [Zhu16] to get a simpler proof that $\Omega(\sqrt{n})$ memory locations, which support only trivial and historyless instructions, are necessary to solve $n$-consensus.

Finally, in Section 9, we show that an unbounded number of memory locations supporting $\{\operatorname{read}()$, write $(1)\}$ or, more generally, $\{\operatorname{read}()$, test-and-set ()$\}$, are necessary and sufficient to solve $n$-consensus, for $n \geq 3$. Furthermore, we show how to reduce the number of memory locations to $O(n \log n)$ when write $(0)$ is also available.

\section{MODEL}

Our model bears similarities to the standard asynchronous shared memory model, albeit with a few important differences. We consider a system of $n \geq 2$ processes that supports some set of deterministic synchronization instructions, $\mathcal{I}$, on a set of identical memory locations. The processes take steps at arbitrary, possibly changing, speeds and may crash at any time. Each step is an atomic invocation of some instruction on some memory location. Scheduling is controlled by an adversary.

The processes can use instructions on the memory locations to simulate (or implement) various objects. An object provides a set of operations which processes can call. Although a memory location together with the supported instructions can be viewed as an object, we do not do so, to emphasize the uniformity requirement that the same set of instructions is supported on all memory locations. 


\begin{tabular}{|c|c|}
\hline Instructions $\mathcal{I}$ & $\mathcal{S P}(\mathcal{I}, n)$ \\
\hline$\{$ read (), test-and-set ()$\},\{$ read (), write $(1)\}$ & $\infty$ \\
\hline$\{\operatorname{read}()$, test-and-set (), reset ()$\},\{$ read (), write $(1)$, write $(0)\}$ & $n-1$ (lower), $O(n \log n)$ (upper) \\
\hline$\{\operatorname{read}()$, write $(x)\}$ & $n-1$ (lower), $n$ (upper) \\
\hline$\{\operatorname{read}(), \operatorname{swap}(x)\}$ & $\Omega(\sqrt{n})$ (lower), $n-1$ (upper) \\
\hline$\{\ell$-buffer-read ()$, \ell$-buffer-write $(x)\}$ & $\left\lceil\frac{n-1}{\ell}\right\rceil$ (lower), $\left\lceil\frac{n}{\ell}\right\rceil$ (upper) \\
\hline $\begin{array}{c}\{\text { read }(), \text { write }(x), \text { increment }()\} \\
\{\operatorname{read}(), \text { write }(x), \text { fetch-and-increment }()\}\end{array}$ & 2 (lower), $O(\log n)$ (upper) \\
\hline$\{$ read-max (), write- $\max (x)\}$ & 2 \\
\hline $\begin{array}{c}\{\text { compare-and-swap }(x, y)\} \\
\{\operatorname{read}(), \text { add }(x)\},\{\text { read }(), \text { multiply }(x)\} \\
\{\text { fetch-and-add }(x)\}\},\{\text { fetch-and-multiply }(x)\}\end{array}$ & 1 \\
\hline
\end{tabular}

Table 1: Space Hierarachy

We consider the problem of solving obstruction-free $m$ valued consensus in such a system. Here, each of the $n$ processes has an input from $\{0,1, \ldots, m-1\}$ and is supposed to output the same input value (called a decision). Obstruction-free means that every process can eventually decide a value if no other process is taking steps at the same time. When $m=n$, we will refer to this problem as $n$ consensus.

In some of our algorithms, we let the processes take snapshots of all memory locations. If the system supports read(), or a similar instruction, then processes can implement an atomic snapshot in an obstruction-free way by using the double collect algorithm in $\left[\mathrm{AAD}^{+}\right.$93]: i.e. they repeatedly collect the values of all locations until they observe two adjacent collects whose values agree in each location.

In our lower bound proofs, we consider solving $n$-consensus when each process starts with an input in $\{0,1\}$ (i.e. binary consensus). We say that a process $p$ is poised to perform a specific instruction on a memory location $r$ at a configuration $C$ if $p$ 's next step in configuration $C$ is to perform that instruction to $r$. Since we consider deterministic algorithms, the next step of a process is uniquely defined at every configuration $C$.

\section{ARITHMETIC INSTRUCTIONS}

Consider a system that supports only $\operatorname{read}()$ and either $\operatorname{add}(x)$ or multiply $(x)$. We show how to solve $n$-consensus using a single memory location in such systems. The idea is to show that we can simulate certain collections of objects that can solve $n$-consensus.

A counter object supports two operations, $\operatorname{read}()$ and increment(), where read() returns the current value of the counter and increment() increments the value of the counter by 1 . In the next lemma, we present a racing counters protocol that bears some similarity to the consensus protocol of Aspnes and Herlihy [AH90].

LEMma 1. It is possible to solve obstruction-free $m$-valued consensus among any number of processes using $m$ counter objects.

Proof. We associate a separate counter $c_{v}$ for each possible input value $v$. Processes that promote a particular value $v$ increment $c_{v}$. (Initially each process promotes its input value.) After performing an increment, a process takes an atomic snapshot of all $m$ counters and promotes the value associated with a counter with the maximum value (breaking ties arbitrarily). When a process observes that some counter $c_{v}$ is more than $n$ larger than all other counters, it returns the value $v$ associated with $c_{v}$. This works because all other processes will increment some counter at most once before next taking a snapshot, where $c_{v}$ will still be the only maximum. From there on, all processes will promote value $v$ and keep incrementing $c_{v}$, which will shortly become large enough for all processes to return $v$. Obstruction-freedom follows because a process running on its own will continue to increment the same counter and, thus, eventually be able to return.

The counters may grow arbitrarily large in the preceding protocol. The next lemma shows that it is possible to overcome this limitation when the counter objects additionally support a decrement() operation, which simply decrements the value of the counter object by 1 . We call such counter objects decrementable.

LEMMA 2. It is possible to solve obstruction-free $m$-valued consensus among any number of processes using $m$ decrementable counter objects, each of which only takes on values in the range $\{0,1, \ldots, 3 n-1\}$.

ProOF. We modify the construction in Lemma 1 slightly by changing what a process does when it wants to increment $c_{u}$ after performing a snapshot to promote the value $u$. Let $c_{v}$ be a counter with the largest value among the other counters (i.e. excluding $c_{u}$, which has the largest value). If $c_{v}<n$, it increments $c_{u}$, as before. If $c_{v} \geq n$, then, instead of incrementing $c_{u}$, it decrements $c_{v}$.

A counter with value 0 is never decremented. This is because, after the last time some process observed it to have value at least $n$, each process will decrement the counter at most once before reading its value again. Similarly, a counter $c_{u}$ never becomes larger than $3 n-1$. After the last time some process observed it to have value less than $2 n$, each process can increment $c_{u}$ at most once before reading its value again. If $c_{u} \geq 2 n$, then either the largest value of the other counters is less than $n$, in which case the process returns without incrementing $c_{u}$, or it is at least $n$, in which case the process decrements a counter with this value, instead of incrementing $c_{u}$.

In the following theorem, we show how to $n$ simulate counters and $n$ decrementable counters.

THEOREM 3. It is possible to solve $n$-consensus using a single memory location that supports only read() and either multiply $(x)$ or add $(x)$. 
Proof. We first give an obstruction-free implementation $n$ counters using a single location in a system with read() and multiply $(x)$ instructions, which proves the claim for this set of instructions, by Lemma 1 . The location is initialized with 1 . For a value $v$, let $p_{v}$ be the $(v+1)$ 'st prime number. A process increments a counter corresponding to $v$ by performing multiply $\left(p_{v}\right)$. A read() instruction returns the value $x$ currently stored in the memory location, which gives a snapshot of all counter values: The value of the counter $c_{v}$ is the exponent of $p_{v}$ in the prime decomposition of $x$.

A similar construction does not work in a system with read() and $\operatorname{add}(x)$ instructions. For example, suppose one counter is incremented by calling $a d d(a)$ and another counter is incremented by calling $a d d(b)$. Then the value $a b$ can be obtained by incrementing the first counter $b$ times or incrementing the second counter $a$ times. However, we can use a single memory location that supports $\{\operatorname{read}(), \operatorname{add}(x)\}$ to implement $n$ different decrementable counters which always have values in $\{0,1, \ldots, 3 n-1\}$, which is also sufficient for solving consensus by Lemma 2. We initialize the location with 0 . We view the value stored in the location as a number written in base $3 n$ and interpret its $i$ 'th least significant digit as the value of counter $c_{i-1}$. To increment $c_{i}$, we perform $\operatorname{add}\left((3 n)^{i}\right)$, to decrement $c_{i}$, we perform $a d d\left(-(3 n)^{i}\right)$, and $\operatorname{read}()$ provides an atomic snapshot of all $n$ counters.

\section{MAX-REGISTERS}

A max-register object [AAC09] supports two operations, write-max $(x)$ and read-max () . The write-max $(x)$ operation sets the value of the max-register to $x$ if $x$ is larger than the current value and read-max() returns the current value of the max-register (which is the largest amongst all values previously written to it). We show that 2 max-registers are necessary and sufficient for solving $n$-consensus.

THEOREM 4. It is not possible to solve n-consensus using a single max-register.

Proof. Consider a solo execution $\alpha$ of the first process that returns 0 , and a solo execution $\beta$ of the second process that returns 1 . We show how to interleave these two executions so that the resulting execution is indistinguishable to both processes from their respective solo executions. Hence, both values will be returned, violating the agreement property of consensus.

To build the interleaved execution, run both processes until there are first poised to perform write-max. Suppose the first process is poised to perform write-max $(a)$ and the second process is poised to perform write-max $(b)$. If $a \leq b$, let the first process take steps until it is next poised to perform write-max or until the end of $\alpha$, if it performs no more write-max operations. Otherwise, let the second process take steps until it is next poised to perform write-max or until the end of $\beta$. Repeat this until one of the processes reaches the end of its execution and then let the other process finish.

THEOREM 5. It is possible to solve $n$-consensus using two max registers.

\section{INCREMENT}

Consider a system that supports only read, write $(x)$, and either fetch-and-increment() or increment(). We prove that it is not possible to solve $n$-consensus in the first (stronger) case using a single memory location and we provide an algorithm in the second (weaker) case, which uses $O(\log n)$ memory locations.

THEOREM 6. It is not possible to solve n-consensus using a single memory location that supports only read (), write $(x)$, and fetch-and-increment () .

Proof. Suppose there is a binary consensus algorithm for two processes, $p$ and $q$, using only 1 memory location. Consider solo executions $\alpha$ and $\beta$ by $p$ with input 0 and input 1 , respectively. Let $\alpha^{\prime}$ be the longest prefix of $\alpha$ that does not contain a write and define $\beta^{\prime}$ analogously. Without the loss of generality, assume that $\beta^{\prime}$ performs at least as many fetch-and-increment instructions as $\alpha^{\prime}$. Let $C$ be the configuration that results from performing $\alpha^{\prime}$ starting from the initial configuration in which $p$ has input 0 and the other process, $q$ has input 1 .

Consider the shortest prefix $\beta^{\prime \prime}$ of $\beta^{\prime}$ in which $p$ performs the same number of fetch-and-increments as it performs in $\alpha^{\prime}$. Let $C^{\prime}$ be the configuration that results from performing $\beta^{\prime \prime}$ starting from the initial configuration in which both $p$ and $q$ have input 1 . Then $q$ must decide 1 in its solo execution $\gamma$ starting from configuration $C^{\prime}$. However, $C$ and $C^{\prime}$ are indistinguishable to process $q$, so it must decide 1 in $\gamma$ starting from configuration $C$. Thus, $p$ cannot have decided yet in configuration $C$, otherwise both 0 and 1 would have been decided.

Therefore, $p$ is poised to perform a write in configuration $C$. Let $\alpha^{\prime \prime}$ be the remainder of $\alpha$, so $\alpha=\alpha^{\prime} \alpha^{\prime \prime}$. The configurations resulting from performing this write starting from $C$ and $C \gamma$ are indistinguishable to $p$, so $p$ also decides 0 starting from $C \gamma$. But in this execution, both 0 and 1 are decided. This is a contradiction.

The following well-known construction converts any algorithm for solving binary consensus to an algorithm for solving $n$-valued consensus [HS12].

LEMMA 7. Consider a system that supports a set of instructions that includes read() and write $(x)$. If it is possible solve obstruction-free binary consensus among $n$ processes using only c memory locations, then it is possible to solve $n$-consensus using only $(c+2) \cdot\left\lceil\log _{2} n\right\rceil-2$ locations.

By Lemma 1, $n$ processes can solve obstruction-free binary consensus using two counter objects and, hence, in this system, using two memory locations. The next result then follows from Lemma 7.

THEOREM 8. It is possible to solve $n$-consensus using only $O(\log n)$ memory locations that support only read () , write $(x)$, and increment () .

\section{6. $\ell$-BUFFERS}

In this section, we consider the instructions $\ell$-buffer-read () and $\ell$-buffer-write $(x)$, for $\ell \geq 1$, which generalize read and write, respectively. Specifically, an $\ell$-buffer-read() instruction returns the last $\ell$ inputs to $\ell$-buffer-write instructions previously applied on the memory location, in order from least recent to most recent.

We consider a system that supports the instruction set $\mathcal{B}_{\ell}=\{\ell$-buffer-read ()$, \ell$-buffer-write $(x)\}$, for some $\ell \geq 1$. 
We call each memory location in such a system an $\ell$-buffer. Note that a 1-buffer is simply a register. For $\ell>1$, an $\ell$-buffer essentially maintains a buffer of the $\ell$ most recent writes to that location and allows them to be read.

In Section 6.1, we show that a single $\ell$-buffer can be used to simulate a powerful history object that can be updated by at most $\ell$ processes. This will immediately allow us to simulate Aspnes and Herlihy's algorithm for $n$-consensus [AH90] and, hence, solve $n$-consensus, using only $\lceil n / \ell\rceil \ell$-buffers. In Section 6.2 , we prove that $\lceil(n-1) / \ell\rceil \ell$-buffers are necessary, which matches the upper bound whenever $n-1$ is not a multiple of $\ell$.

\subsection{Simulations Using $\ell$-Buffers}

A history object $H$ supports two operations, get-history() and append $(x)$, where get-history () returns the sequence of all values appended to $H$ by prior append operations, in order. We first show that, using a single $\ell$-buffer $B$, we can simulate a history object $H$ that supports at most $\ell$ different appenders, but arbitrarily many readers.

LEMma 9. A single $\ell$-buffer can simulate a history object on which at most $\ell$ different processes can perform append $(x)$ and any number of processes can perform get-history ().

Proof. Without loss of generality, assume that no value is appended to $H$ more than once. This can be achieved by having a process include its process identifier and a sequence number along with the value that it wants to append.

In our implementation, $B$ is initially $\perp$ and each value written to $B$ is of the form $(\mathbf{h}, x)$, where $\mathbf{h}$ is a history of appended values and $x$ is a single appended value.

To get-history() from $H$, a process simply performs an $\ell$-buffer-read of $B$ to obtain a vector $\left(a_{1}, \ldots, a_{\ell}\right)$. The operation is linearized at this step. If $\left(a_{1}, \ldots, a_{\ell}\right)=(\perp, \ldots, \perp)$, then the empty sequence is returned. Otherwise, let $k$ be the smallest integer such that $a_{k} \neq \perp$. It follows that, for $k \leq i \leq \ell, a_{i}=\left(\mathbf{h}_{i}, x_{i}\right)$, where $\mathbf{h}_{i}$ is a history and $x_{i}$ is an appended value. If $k>1$, then $\left(x_{k}, x_{k+1}, \cdots, x_{\ell}\right)$ is returned. Now suppose $k=1$. Let $\mathbf{h}$ be the longest history amongst $\mathbf{h}_{1}, \ldots, \mathbf{h}_{\ell}$. If $\mathbf{h}$ contains $x_{1}$, then $\mathbf{h}^{\prime} \cdot\left(x_{1}, \ldots, x_{\ell}\right)$ is returned, where $\mathbf{h}^{\prime}$ is the prefix of $\mathbf{h}$ up to, but not including, $x_{1}$. If $\mathbf{h}$ does not contain $x_{1}$, then $\mathbf{h} \cdot\left(x_{1}, \ldots, x_{\ell}\right)$ is returned.

To append $(x)$ to $H$, a process first performs a get-history () on $H$ to obtain a history $\mathbf{h}$ and then performs $\ell$-buffer-write on $B$ with value $(\mathbf{h}, x)$. The operation is linearized at this $\ell$-buffer-write step.

We claim that every get-history() operation $X$ returns the sequence of inputs to all append operations on $H$ linearized before the linearization point of $X$, in order from least recent to most recent. The proof is by induction on the number of append operations on $H$ linearized before $X$. Let $R$ be the $\ell$-buffer-read step in $X$, which is the linearization point of $X$, and let $\left(a_{1}, \ldots, a_{\ell}\right)$ be the vector returned by $R$.

If there are no append operations linearized before $R$, then there are no $\ell$-buffer-write steps before $R$. It follows that $\left(a_{1}, \ldots, a_{\ell}\right)=(\perp, \ldots, \perp)$ and $X$ returns the empty sequence, as required.

Now, suppose that there is at least one append operation linearized before $R$. Let $k$ be the smallest integer such that $a_{k} \neq \perp$. For $k \leq i \leq \ell, a_{i}=\left(\mathbf{h}_{i}, x_{i}\right)$, where $\mathbf{h}_{i}$ is a history returned by a get-history () operation $X_{i}$ on $H$ which had strictly fewer append operations linearized before it than $X$.
If $k>1$, then there are only $\ell-k+1$ occurrences of $\ell$-buffer-write before $R$. Since each append operation is linearized at its $\ell$-buffer-write, only $\ell-k+1$ append instructions are linearized before $X$ and $x_{k}, \ldots, x_{\ell}$ are the values appended by these $\ell-k+1$ append operations, in order from least recent to most recent. In this case, $\left(x_{k}, x_{k+1}, \ldots, x_{\ell}\right)$ is returned, as required.

Now suppose $k=1$. Let $\mathbf{h}$ be the longest history amongst $\mathbf{h}_{1}, \ldots, \mathbf{h}_{\ell}$, let $m$ be such that $\mathbf{h}_{m}=\mathbf{h}$, and let $W$ be the $\ell$-buffer-write which wrote $x_{1}$ to $B$. There are two cases to consider.

Case 1: $\mathbf{h}$ contains $x_{1}$. By the induction hypothesis, $X_{m}$ returns the history of all values appended to $H$ by append operations linearized before $X_{m}$, in order from least recent to most recent. By definition, $x_{1}, \ldots, x_{\ell}$ are the last $\ell$ values appended to $H$ prior to $R$, in order. Since $\mathbf{h}$ contains $x_{1}$, it must also contain all values appended to $H$ prior to $x_{1}$. It follows that $\mathbf{h}^{\prime} \cdot\left(x_{1}, \ldots, x_{\ell}\right)$ is the correct return value, where $\mathbf{h}^{\prime}$ is the prefix of $\mathbf{h}$ up to, but not including, $x_{1}$.

Case 2 : $\mathbf{h}_{1}$ does not contain $x_{1}$. Since $\mathbf{h}_{i}$ is a prefix of $\mathbf{h}$, it does not contain $x_{1}$, either. By the induction hypothesis, $W$ occurs after $X_{1}, \ldots, X_{\ell}$. Thus, the append operations which performed $X_{1}, \ldots, X_{\ell}$ are all concurrent. In particular, $W$ occurs in each of their execution intervals. It follows that these append operations are all by different processes. Since there are at most $\ell$ updating processes, there is no append operation linearized between $X_{m}$ and $W$. Therefore, $\mathbf{h}$ contains all values appended to $H$ prior to $W$. It follows that $\mathbf{h} \cdot\left(x_{1}, \ldots, x_{\ell}\right)$ is the correct return value.

In fact, this lemma allows us to simulate any object that supports at most $\ell$ updating processes using only a single $\ell$ buffer. This is because the state of an object is determined by the history of the non-trivial operations performed on it. In particular, we can simulate an array of $\ell$ single-writer registers using a single $\ell$-buffer.

LEMma 10. A single $\ell$-buffer can simulate $\ell$ single-writer registers.

Thus, we can use $\left\lceil\frac{n}{\ell}\right\rceil \ell$-buffers to simulate $n$ single-writer registers. This, in turn, allows us to simulate the $n$-consensus algorithm of Aspnes and Herlihy [AH90].

THEOREM 11. It is possible to solve $n$-consensus using only $\lceil n / \ell\rceil \ell$-buffers.

\subsection{A Lower Bound}

In this section, we show a lower bound on the number of memory locations necessary for solving obstruction-free binary consensus among $n \geq 2$ processes.

A location $r$ is covered by a process $p$ in some configuration, if $p$ is poised to perform $\ell$-buffer-write on $r$. An $\ell$-buffer is $k$-covered by a set of processes $\mathcal{P}$ in some configuration, if there are exactly $k$ processes in $\mathcal{P}$ that cover it. A configuration is at most $k$-covered by $\mathcal{P}$, if no $\ell$-buffer in the system is $k^{\prime}$-covered by $\mathcal{P}$ in this configuration, for any $k^{\prime}>k$.

Let $\mathcal{Q}$ be a set of processes, each of which is poised to perform $\ell$-buffer-write in some configuration $C$. A block write by $\mathcal{Q}$ from $C$ is an execution, starting at $C$, in which each process in $\mathcal{Q}$ takes exactly one step. If a block write is performed that includes $\ell$ different $\ell$-buffer-write instructions to some $\ell$-buffer, then any process that performs $\ell$-buffer-read 
on that $\ell$-buffer immediately afterwards, gets the same result (and ends up in the same state) regardless of the value of that $\ell$-buffer in $C$.

We say that a set of processes $\mathcal{P}$ can decide $v \in\{0,1\}$ from a configuration $C$ if there exists a $\mathcal{P}$-only execution from $C$ in which $v$ is decided. If $\mathcal{P}$ can decide both 0 and 1 from $C$, then $\mathcal{P}$ is bivalent from $C$. If $\mathcal{P}$ can decide $v$, but not $\bar{v}$, from $C$, then $\mathcal{P}$ is $v$-univalent from $C$. Note that, because the system is deterministic, each process $p$ has only one solo execution starting from $C$ that decides a value. Thus $\{p\}$ is either 0-univalent or 1-univalent from $C$.

To obtain the lower bound, we extend the proof of the $n-1$ lower bound on the number of registers required for solving $n$-process consensus [Zhu16], borrowing intuition about reserving executions from the $\Omega(n)$ lower bound for anonymous consensus [Gel15]. The following auxiliary lemmas are largely unchanged from [Zhu16]. The main difference is that we only perform block writes on $\ell$-buffers that are $\ell$-covered by $\mathcal{P}$.

LEMMA 12. There is an initial configuration from which the set of all processes in the system is bivalent.

Lemma 13. Let $R$ be a set of locations that are $\ell$-covered by $\mathcal{P}$ in $C$, let $\mathcal{Q} \subseteq \mathcal{P}$ be the set of the $\ell \cdot|R|$ processes that cover $R$, and let $\beta$ be a block write from $C$ by $\mathcal{Q}$. Suppose some process $q \in \mathcal{Q}$ can decide some value $v \in\{0,1\}$ from configuration $C \beta$ and $\gamma$ is a p-only execution from $C$ by a process $p \notin \mathcal{Q}$ in which $\bar{v}$ is decided. Let $\gamma^{\prime}$ be the longest prefix of $\gamma$ such that $q$ can decide $v$ from $C \gamma^{\prime} \beta$. Then, in configuration in $C \gamma^{\prime}, p$ covers a location not in $R$ and $\{p, q\}$ is bivalent from $C \gamma^{\prime} \beta$.

The next result says that if a set of processes is bivalent in some configuration, then it is possible to reach a configuration from which some process can decide 0 and another process can decide 1 . It does not depend on what instructions are supported by the memory.

Lemma 14. Suppose a set $U$ of at least two processes is bivalent from configuration $C$. Then it is possible to reach, via a $U$-only execution from $C$, a configuration $C^{\prime}$ in which there are processes $p_{0}, p_{1} \in U$, such that for each $v \in\{0,1\}$, $p_{v}$ can decide $v$ from $C^{\prime}$.

An induction similar to [Zhu16] allows the processes to reach a configuration that is at most $\ell$-covered by a set of processes $\mathcal{R}$, while another process $z \notin \mathcal{R}$ covers a location that is not $\ell$-covered by $\mathcal{R}$. This implies that the configuration is also at most $\ell$-covered by $\mathcal{R} \cup\{z\}$, allowing the inductive step to go through.

Lemma 15. Let $C$ be a configuration and let $\mathcal{P}$ be a set of $n \geq 2$ processes. If $\mathcal{P}$ is bivalent from $C$, then there is a $\mathcal{P}$-only execution $\alpha$ and a pair of processes $\mathcal{Q} \subseteq \mathcal{P}$ such that $\mathcal{Q}$ is bivalent from $C \alpha$ and $C \alpha$ is at most $\ell$-covered by the remaining processes $\mathcal{P}-\mathcal{Q}$.

Finally, we can prove the main theorem.

THEOREM 16. Consider a memory consisting of $\ell$-buffers. Then any algorithm for solving obstruction-free binary consensus for $n$ processes uses at least $\lceil(n-1) / \ell\rceil$ locations.
Proof. Consider an obstruction-free binary consensus algorithm. Let $C$ be an initial configuration from which the set of all $n$ processes, $\mathcal{P}$, is bivalent. Such a configuration exists by Lemma 12 . Lemma 15 implies there is a reachable configuration $C$ and a pair of processes $\mathcal{Q} \subseteq \mathcal{P}$ that are bivalent from $C$. Furthermore, $C$ is at most $\ell$-covered by the remaining processes $\mathcal{R}$. By the pigeonhole principle, $\mathcal{R}$ covers at least $\lceil(n-2) / \ell\rceil$ different locations. If $\lceil(n-2) / \ell\rceil<\lceil(n-1) / \ell\rceil$, then $n-2$ is a multiple of $\ell$ and every location covered by $\mathcal{R}$ is in fact $\ell$-covered by $\mathcal{R}$. By Lemma 13 , since $\mathcal{Q}$ is bivalent from $C$, we can use a process in $\mathcal{Q}$ to cover a location not $\ell$-covered by $\mathcal{R}$. Hence, there are at least $\lceil(n-2) / \ell\rceil+1=\lceil(n-1) / \ell\rceil$ locations.

The lower bound can be easily generalized to consensus algorithms that satisfy nondeterministic solo termination [FHS98]. It can also be extended to a heterogeneous setting, where memory locations need not be identical, for example, $\ell$-buffers for possibly different values of $\ell$. For this, we need to extend the definition of at most $\ell$-covered to, instead, require each $\ell$-buffer to be covered by at most $\ell$ processes, for all values of $\ell$. Then we consider block writes to a set of locations containing $\ell$ different $\ell$-buffer-write operations to each $\ell$-buffer in the set. The general result is that, for any algorithm which solves consensus for $n$ processes and satisfies non-deterministic solo termination, the sum of capacities of all buffers must be at least $n-1$.

The lower bound also applies to systems in which the output of every non-trivial instruction on a memory location does not depend on the value of that location and the output of any trivial instruction is a function of the sequence of the preceding $\ell$ non-trivial instructions performed on the location. This is because such instructions can be implemented by $\ell$-buffer-read and $\ell$-buffer-write instructions.

\section{MULTIPLE ASSIGNMENT}

With $m$-register multiple assignment, we can atomically write to $m$ locations. This plays an important role in [Her91], as $m$-register multiple assignment can used to solve wait-free consensus for $2 m-2$ processes, but not for $2 m-1$ processes.

In this section, we explore whether multiple assignment could improve the space complexity of solving obstructionfree consensus. A practical motivation to this question is that obstruction-free multiple assignment can be easily implemented using s simple transaction.

We prove a lower bound that is similar to the lower bound in Section 6.2. Suppose $\ell$-buffer-read () and $\ell$-buffer-write $(x)$ instructions are supported on every memory location in a system and, for any subset of locations, we are allowed to atomically perform one $\ell$-buffer-write instruction per location. Then $\lceil n / 2 \ell\rceil$ locations are necessary for $n$ processes to solve obstruction-free consensus. As in Section 6.2, this result can be generalized to nondeterministic solo termination, different sets of instructions, and heterogeneous settings.

The main difficulty in this lower bound is proving an analogue of Lemma 13. In the absence of multiple assignment, if $\beta$ is a block $\ell$-buffer-write to a set of $\ell$-covered locations $R$ and $\delta$ is an $\ell$-buffer-write to a location not in $R$, then $\beta$ and $\delta$ trivially commute (in the sense that the resulting configurations are indistinguishable to all processes). However, a multiple assignment $\delta$ may now atomically $\ell$-buffer-write to many locations, including some in $R$. Thus, it is now possible for processes to distinguish between $\beta \delta$ and $\delta \beta$. Us- 
ing a careful combinatorial argument, we show how to perform a block of multiple assignments $\beta^{1} \beta^{2}$, such that, in $\beta^{i}$, $\ell$-buffer-write is invoked exactly $\ell$ times for each location in $R$, and is never invoked for any location not in $R$. Given this, we can show that $\beta^{1} \delta \beta^{2}$ and $\delta \beta^{1} \beta^{2}$ are indistinguishable to all processes, which is enough to prove an analogue of Lemma 13.

First, we define the notion of covering in this setting. We say that a process $p$ covers location $r$ in a configuration $C$, when the next step of $p$ in $C$ is a multiple assignment that involves an $\ell$-buffer-write to $r$. The next definition is key to our proof. A $k$-packing of a set of processes $\mathcal{P}$ in some configuration $C$ is a function $\pi$ mapping each process in $\mathcal{P}$ to some memory location it covers such that no location $r$ has more than $k$ processes mapped to it (i.e., $\left|\pi^{-1}(r)\right| \leq k$ ). When $\pi(p)=r$ we say that $\pi$ packs $p$ in $r$. A $k$-packing may not always exist, or there may be many, depending on the configuration, the set of processes, and the value of $k$. A location $r$ is fully $k$-packed by $\mathcal{P}$ in configuration $C$, if a $k$-packing of $\mathcal{P}$ exists in $C$ and all such $k$-packings pack exactly $k$ processes in $r$.

Suppose that, in some configuration, there are two $k$ packings of the same set of processes such that the first packs more processes in some location $r$ than the second. We show there is a location $r^{\prime}$ in which the first packing packs fewer processes than the second and a $k$-packing that pack one less process to location $r$, one more process to location $r^{\prime}$ and the same number of processes to all other locations, as compare to the first packing. This iterative procedure has some resemblance to cuckoo hashing [PR01]. The proof relies on existence of a certain Eulerian path in a multigraph that we build to represent these two $k$-packings.

Lemma 17. Suppose $g$ and $h$ are two $k$-packings of the same set of processes $\mathcal{P}$ in some configuration $C$ and $r_{1}$ is a location such that $g$ packs more processes in $r_{1}$ than $h$ does (i.e., $\left|g^{-1}\left(r_{1}\right)\right|>\left|h^{-1}\left(r_{1}\right)\right|$ ). Then, there exists a sequence of locations $r_{1}, r_{2}, \ldots, r_{t}$ and a sequence of distinct processes $p_{1}, p_{2}, \ldots, p_{t-1}$, such that $h$ packs more processes in $r_{t}$ than $g$, (i.e., $\left|h^{-1}\left(r_{t}\right)\right|>\left|g^{-1}\left(r_{t}\right)\right|$ ) and, for $1 \leq i \leq t-1, g\left(p_{i}\right)=$ $r_{i}$ and $h\left(p_{i}\right)=r_{i+1}$.

Proof. Consider a multigraph with one node for each memory location in the system and one directed edge labelled by $p$, from node $g(p)$ to node $h(p)$, for each process $p \in \mathcal{P}$. Therefore, the in-degree of a node $v$ is equal to $\left|h^{-1}(v)\right|$, which is the number of processes that are packed into memory location $v$ by $h$, and the out-degree of node $v$ is equal to $\left|g^{-1}(v)\right|$, which is the number of processes that are packed in $v$ by $g$.

Now, consider any maximal Eulerian path in this multigraph starting from node $r_{1}$. This path follows a sequence of edges for as long as possible without repeating any edge. Let $r_{1}, \ldots, r_{t}$ be the sequence of nodes visited (which may contain repetitions) and let $p_{i}$ be the labels of the traversed edges, in order. Then $g\left(p_{i}\right)=r_{i}$ and $h\left(p_{i}\right)=r_{i+1}$ for $1 \leq i \leq t-1$. Since the path is Eulerian, the labels of the edges are distinct, which, in turn, guarantees that these sequences are finite. Finally, by maximality, the last node in the sequence must have more incoming than outgoing edges, so $\left|h^{-1}\left(r_{t}\right)\right|>\left|g^{-1}\left(r_{t}\right)\right|$.

COROLlary 18. Let the $k$-packings $g$ and $h$ and the sequences $r_{i}$ and $p_{i}$ be defined as in Lemma 1\%. For $1 \leq j<t$, there exists a $k$-packing $g^{\prime}$, such that $g^{\prime}$ packs one less process than $g$ in $r_{j}$, one more process than $g$ in $r_{t}$, and the same number of processes as $g$ in all other locations.

PROOF. We construct $g^{\prime}$ from $g$ by re-packing each process $p_{i}$ from $r_{i}$ to $r_{i+1}$ for all $j \leq i<t$. Then $g^{\prime}\left(p_{i}\right)=r_{i+1}$ for $j \leq i<t$ and $g^{\prime}(p)=g(p)$ for all other processes $p$. Notice that $p_{i}$ covers $r_{i+1}$, since $h\left(p_{i}\right)=r_{i+1}$ and $h$ is a $k$-packing.

As compared to $g, g^{\prime}$ packs one less process in $r_{j}$, one more process in $r_{t}$, and the same number of processes in every other location. Since $h$ is a $k$-packing, it packs at most $k$ processes in $r_{t}$. Because $g$ is a $k$-packing that packs less processes in $r_{t}, g^{\prime}$ is also a $k$-packing.

Let $\mathcal{Q}$ be a set of processes, each of which is poised to perform a multiple assignment in some configuration $C$. A block multi-assignment by $\mathcal{Q}$ from $C$ is an execution interval starting at $C$, in which each process in $\mathcal{Q}$ takes exactly one step. Consider some configuration $C$, a set of processes $\mathcal{P}$, and a $2 \ell$-packing $\pi$ of $\mathcal{P}$ in $C$. Let $R$ be the set of all locations that are fully $2 \ell$-packed by $\mathcal{P}$ in $C$. By definition, $\pi$ packs exactly $2 \ell$ processes from $\mathcal{P}$ in each location $r \in R$.

Suppose $\mathcal{Q}_{1}, \mathcal{Q}_{2} \subseteq \mathcal{P}$ is a partition of all processes that are packed in $R$ by $\pi$, such that for each location $r \in R$, exactly $\ell$ packed processes belong to $\mathcal{Q}_{1}$ and the remaining $\ell$ packed processes belong to $\mathcal{Q}_{2}$. Let $\beta_{i}$ be a block multi-assignment by $\mathcal{Q}_{i}$ for $i \in\{1,2\}$.

Notice that, for any location $r \in R$, after $\beta_{1}$ or $\beta_{2}$, the outcome of any subsequent $\ell$-buffer-read on $r$ does not depend on multiple assignments that occurred prior to the block multi-assignment. Moreover, we can prove the following crucial property about these block multi-assignments to fully packed locations.

Lemma 19. Neither $\beta_{1}$ nor $\beta_{2}$ involves an $\ell$-buffer-write to a location outside of $R$.

Proof. Assume the contrary. Let $q \in \mathcal{Q}_{1} \cup \mathcal{Q}_{2}$ be a process with $\pi(q) \in R$, such that $q$ also covers some location $r_{1} \notin R$ in $C$. It must be the case that $\pi$ packs exactly $2 \ell$ processes in $r_{1}\left(\left|\pi^{-1}\left(r_{1}\right)\right|=2 \ell\right)$. Otherwise, re-packing $q$ in $r_{1}$ instead of $\pi(q)$ leads to another $2 \ell$-packing that does not pack exactly $2 \ell$ processes in $\pi(q) \in R$, contradicting the definition of a fully $2 \ell$-packed location.

Since $R$ is the set of all fully $2 \ell$-packed locations, there exists a $2 \ell$-packing $h$, which packs strictly less than $2 \ell$ processes in $r_{1}$. Applying Lemma 17 for $2 \ell$-packings $\pi$ and $h$, we get a sequence of locations $r_{1}, \ldots, r_{t}$ and a sequence of processes $p_{1}, \ldots p_{t-1} .\left|\pi^{-1}\left(r_{t}\right)\right|<\left|h^{-1}\left(r_{t}\right)\right|$, and since $h$ is a 2 $\ell$-packing, we must have that $\left|\pi^{-1}\left(r_{t}\right)\right|<2 \ell$, i.e. $\pi$ packs strictly less than $2 \ell$ processes in $r_{t}$ in $C$. We consider two cases.

First, assume that $q$ does not occur in the sequence, i.e. $\forall i: p_{i} \neq q$. We know that $\left|\pi^{-1}\left(r_{1}\right)\right|=2 \ell$ and $\left|\pi^{-1}\left(r_{t}\right)\right|<$ $2 \ell$, implying that the locations $r_{1}$ and $r_{t}$ must be different. This allows us to apply Corollary 18 for the whole sequence with $j=1$. We get a $2 \ell$-packing $\pi^{\prime}$ that packs less than $2 \ell$ processes in $r_{1}$, and exactly $2 \ell$ processes in each of the fully $2 \ell$-packed locations $R$, including $\pi(q)$. Moreover, we did not re-pack process $q$, so $\pi^{\prime}(q)=\pi(q)$. Hence, modifying $\pi^{\prime}$ by re-packing $q$ in $r_{1}$ instead of $\pi^{\prime}(q)$ again leads to a $2 \ell$-packing that does not pack exactly $2 \ell$ processes in a location in $R$, a contradiction.

Now, assume that $q=p_{s}$, for some $s$. By the properties of our sequences, we know $r_{s}=\pi\left(p_{s}\right)=\pi(q) \in R$, and since 
this location is fully $2 \ell$-packed in configuration $C$ we have $\left|\pi^{-1}\left(r_{s}\right)\right|=2 \ell$. Similar to the first case, since $\left|\pi^{-1}\left(r_{t}\right)\right|<$ $2 \ell$ in $C$, locations $r_{s}$ and $r_{t}$ must be different and we now apply Corollary 18 with $j=s$. We get a $2 \ell$-packing $\pi^{\prime \prime}$ that packs less than $2 \ell$ processes in location $r_{s} \in R$. This is a contradiction with the definition of a fully $2 \ell$-packed location, completing the proof.

We can now prove a lemma that replaces Lemma 13 in the main argument.

LEMma 20. Suppose $C \beta^{1} \beta^{2}$ is v-deciding for $\mathcal{Q}_{1} \cup \mathcal{Q}_{2}$ for some $v \in\{0,1\}$. If $\gamma$ is a solo execution from $C$ by a process $p \notin \mathcal{Q}_{1} \cup \mathcal{Q}_{2}$ in which $\bar{v}$ is decided, and $\gamma^{\prime}$ is the longest prefix of $\gamma$ such that $C \gamma^{\prime} \beta^{1} \beta^{2}$ is v-deciding for $\mathcal{Q}_{1} \cup \mathcal{Q}_{2}$, then $p$ is poised to perform a multiple assignment which contains an $\ell$-buffer-write to a location not in $R$ in $C \gamma^{\prime}$ and the configuration $C \gamma^{\prime} \beta^{1}$ is bivalent for $\mathcal{Q}_{1} \cup \mathcal{Q}_{2} \cup\{p\}$.

Proof. Let $\delta$ be the next step by $p$ in $\gamma$ after $\gamma^{\prime}$. Since $C \gamma^{\prime} \beta^{1} \beta^{2}$ is $v$-deciding for $\mathcal{Q}_{1} \cup \mathcal{Q}_{2}$, but $C \gamma^{\prime} \delta \beta^{1} \beta^{2}$ is $\bar{v}$ univalent for $\mathcal{Q}_{1} \cup \mathcal{Q}_{2}, \delta$ must be a multiple assignment which includes an $\ell$-buffer-write to a location not in $R$. (If $\delta$ is a read, the resulting configurations would be indistinguishable. Similarly, if $\delta$ is a multiple assignment containing only $\ell$-buffer-write's to locations in $R$, then no process in $\mathcal{Q}_{1} \cup \mathcal{Q}_{2}$ can observe a difference due to the block writes $\beta^{1} \beta^{2}$.)

We claim that the configuration $C \gamma^{\prime} \beta^{1} \delta \beta^{2}$ is indistinguishable from $C \gamma^{\prime} \delta \beta^{1} \beta^{2}$ to $\mathcal{Q}_{1} \cup \mathcal{Q}_{2}$. Indeed, for each location $r \in R$, since $\left|\pi^{-1}(r) \cap \mathcal{Q}_{i}\right|=\ell$ (that is, $r$ is covered by exactly $\ell$ processes in $\mathcal{Q}_{i}$ ), the contents in location $r$ is the same in $C \gamma^{\prime} \delta \beta^{1} \beta^{2}$ as it is in $C \gamma^{\prime} \beta^{1} \delta \beta^{2}$. On the other hand, for each location $r \notin R$, by Lemma 19, neither $\beta^{1}$ nor $\beta^{2}$ performs an $\ell$-buffer-write to $r$, thus, the contents in location $r$ is the same in $C \gamma^{\prime} \delta \beta^{1} \beta^{2}$ as it is in $C \gamma^{\prime} \beta^{1} \delta \beta^{2}$.

Therefore, $C \gamma^{\prime} \beta^{1} \delta \beta^{2}$ is $\bar{v}$-univalent for $\mathcal{Q}_{1} \cup \mathcal{Q}_{2}$, hence $C \gamma^{\prime} \beta^{1}$ is $\bar{v}$-deciding for $\mathcal{Q}_{1} \cup \mathcal{Q}_{2} \cup\{p\}$. Moreover, $C \gamma^{\prime} \beta^{1} \beta^{2}$ is $v$-deciding for $\mathcal{Q}_{1} \cup \mathcal{Q}_{2}$ by definition of $\gamma^{\prime}$, thus $C \gamma^{\prime} \beta^{1}$ is $v$-deciding for $\mathcal{Q}_{1} \cup \mathcal{Q}_{2} \cup\{p\}$. We have thus established the desired bivalency of $C \gamma^{\prime} \beta^{1}$, completing the proof.

Using these tools, we can prove the following analogue of Lemma 15:

Lemma 21. Let $C$ be a configuration and let $\mathcal{P}$ be a set of $n \geq 2$ processes. If $\mathcal{P}$ is bivalent from $C$, then there is a $\mathcal{P}$-only execution $\alpha$ and a pair of processes $\mathcal{Q} \subseteq \mathcal{P}$ such that $\mathcal{Q}$ is bivalent from $C \alpha$ and there is a $2 \ell$-packing $\pi$ of the remaining processes $\mathcal{P}-\mathcal{Q}$ in $C \alpha$.

We can now prove the main theorem.

THEOREM 22. Consider a memory consisting of $\ell$-buffers. If the processes can atomically $\ell$-buffer-write to any subset of the $\ell$-buffers, then any algorithm for solving obstruction consensus for $n$ processes needs at least $\lceil(n-1) / 2 \ell\rceil$ space.

Proof. Consider an obstruction-free binary consensus algorithm. Let $C$ be an initial configuration from which the set of all $n$ processes, $\mathcal{P}$, is bivalent. Such a configuration exists by Lemma 12. Lemma 21 implies there is a reachable configuration $C$ and a pair of processes $\mathcal{Q} \subseteq \mathcal{P}$ that are bivalent from $C$. Furthermore, there is a $2 \ell$-packing $\pi$ of the remaining processes $\mathcal{R}=\mathcal{P}-\mathcal{Q}$ in $C$. Let $R$ be the set of all locations that are covered by $\mathcal{R}$. By the pigeonhole principle, $|R| \geq\lceil(n-2) / 2 \ell\rceil$ different locations. If $|R|<\lceil(n-1) / 2 \ell\rceil$, then $|R|=\lceil(n-2) / 2 \ell\rceil, n-2$ is a multiple of $2 \ell$, and every location in $R$ is fully $2 \ell$-packed by $\mathcal{R}$. By Lemma 20 , since $\mathcal{Q}$ is bivalent from $C$, we can use a process in $\mathcal{Q}$ to cover a location not fully $2 \ell$-packed by $\mathcal{R}$. Hence, there are at least $\lceil(n-2) / 2 \ell\rceil+1=\lceil(n-1) / 2 \ell\rceil$ locations.

\section{SWAP AND READ}

It is possible to solve $n$-consensus using $n-1$ memory locations that support only $\operatorname{read}()$ and $\operatorname{swap}(x)$ The idea is to modify the anonymous algorithm of [Zhu15], which uses $n$ locations supporting read () and write $(x)$. In that algorithm, processes obtain a snapshot of all $n$ values by repeatedly performing collect. If all $n$ memory locations have the same (non- $\perp$ ) contents, the process returns the value each of them contains. Otherwise, it overwrites one location and repeats the same sequence of steps again. After some process $p$ returns, each of the remaining $n-1$ processes may overwrite one location before taking a snapshot. This ensures that that the value $p$ decided is not lost, since it is contained in at least one location.

In the modified algorithm, instead of collecting the contents from $n$ locations, a process collects the contents from $n-1$ locations and also uses the result of its last swap. After some process $p$ returns, if all $n-1$ remaining processes perform swap on different locations, the result of each of their swap operations is the contents of each location seen by $p$ in its last snapshot. Otherwise, as in the original algorithm, the value $p$ decided is contained in at least one location.

A lower bound of $\Omega(\sqrt{n})$ locations when the system only supports swap andread instructions appears in [FHS98]. We can also adapt the recent proof of [Zhu16], which corresponds to our proof for buffered read-write instructions with capacity $\ell=1$, to get a simpler proof of the $\Omega(\sqrt{n})$ lower bound

Only the proof of Lemma 15 needs to change. It must change because the processes perform block swaps instead of block writes, and can detect the steps by process $z$. To account for this, in the inductive step from $i$ to $i+1$ locations, we consider a set $Z$ of $i+1$ processes instead of the single process $z$. We still perform the block swaps and reach new configurations. If, in any such configuration, two processes in $Z$ cover the same location. then steps are allocated to one of them, until it covers a location not covered by any other process in $Z$. This is repeated until all processes in $Z$ cover $i+1$ different locations. Counting the number of extra processes used in the argument leads to the lower bound of $\Omega(\sqrt{n})$ locations.

\section{TEST-AND-SET AND READ}

Consider a system that supports only test-and-set() and $\operatorname{read}()$. If there are only 2 processes, then it is possible to solve wait-free consensus using a single memory location. However, we claim that any algorithm for solving obstruction-free binary consensus among $n \geq 3$ processes must use an unbounded number of memory locations. The key is to prove the following analogue of Lemma 13.

Lemma 23. Let $P$ be a set of at least 3 processes and let $C$ be a configuration. If $P$ is bivalent from $C$, then, for every $k \geq 0$, there exists a P-only execution $\alpha_{k}$ from $C$ such that $P$ is bivalent from $C \alpha_{k}$ and at least $k$ locations have been set to 1 in $C \alpha_{k}$. 
Proof. By induction on $k$. The base case is when $k=0$ and is trivial. Given $\alpha_{k}$, we show how to construct $\alpha_{k+1}$. By Lemma 14, there is a $P$-only execution $\varphi$ from $C \alpha_{k}$ and a pair of processes, $p_{0}$ and $p_{1}$, that can decide 0 and 1 , respectively, from $C \alpha_{k} \varphi$. Pick $z \in P-\left\{p_{0}, p_{1}\right\}$. Since $\left\{p_{0}, p_{1}\right\}$ is bivalent from $C \alpha_{k} \varphi$, we may run $z$ until it first performs a test-and-set() on a memory location, call it $r$, that had not been set to 1 . Let $\beta$ denote this execution. If $C \alpha_{k} \varphi \beta$ is bivalent from $\left\{p_{0}, p_{1}\right\}$, then we are done with $\alpha_{k+1}=\alpha_{k} \varphi \beta$. So, without loss of generality, suppose $\left\{p_{0}, p_{1}\right\}$ is 0 -univalent from $C \alpha_{k} \varphi \beta$. Let $\psi$ be the longest prefix of $p_{1}$ 's solo terminating execution from $C \alpha_{k} \varphi$ such that $C \alpha_{k} \varphi \psi \beta$ is 0univalent for $\left\{p_{0}\right\}$. It follows that, in configuration $C \alpha_{k} \varphi \psi$, the set $\beta^{\prime}$ that $p_{1}$ is poised to perform is a test-and-set() on an unset memory location. Note that this location is not $r$, since, otherwise, $C \alpha_{k} \varphi \psi \beta^{\prime} \beta$ is indistinguishable from $C \alpha_{k} \varphi \psi \beta$ to $p_{0}$. Therefore, $C \alpha_{k} \varphi \psi \beta \beta^{\prime}$ is indistinguishable from $C \alpha_{k} \varphi \psi \beta^{\prime} \beta$ to $p_{0}$. Let $\alpha_{k+1}=\alpha_{k} \varphi \psi \beta$. It follows that $\left\{p_{0}, p_{1}\right\}$ is bivalent from $C \alpha_{k+1}$. Furthermore, $\beta$ includes a test-and-set() to a location that was not already set to 1 and $C \alpha_{k}$ had at least $k$ locations set to $1, C \alpha_{k+1}$ has at least $k+1$ locations set to 1 .

By Lemma 12, there is an initial configuration from which the set of all processes in the system is bivalent. It follows that we may repeatedly apply the preceding lemma to show that any binary consensus algorithm for $n \geq 3$ processes uses an unbounded number of locations.

TheOREM 24. For $n \geq 3$, it is not possible to solve $n$ consensus using a bounded number of memory locations supporting only read() and test-and-set().

There is an algorithm for obstruction-free binary consensus that uses an unbounded number of binary registers, initially all set to 0 , and only performs write(1) [GR05]. The idea is to simulate a counter using an unbounded number of binary registers and then to run the racing counters algorithm presented in Lemma 1. In their algorithm, there are two unbounded tracks on which processes (with preference 0 and 1 , respectively) race, flipping 0 's to 1 's one by one on their respective tracks to indicate progress. A process changes its preference if it sees that the number of 1's on its side is less than the number of 1's on the other side. Once a process sees that its side is at least 2 ahead of the other side, it decides its current preference.

It is possible to generalize this algorithm to solve $n$-valued consensus by having $n$ race tracks, where register $j$ is used for track $k$ whenever $j \equiv k \bmod n$. Since test-and-set() is at least as powerful as write(1), we get the following result.

TheOREM 25. It is possible to solve $n$-consensus using only an unbounded number of memory locations supporting only read() and either write(1) or test-and-set().

Now, suppose we can also reset() a memory location from 1 to 0 or perform write $(0)$. Then we can solve $n$-consensus using only $O(n \log n)$ locations by combining an existing binary consensus algorithm that uses only $2 n$ binary registers [Bow11] with Lemma 7. There is a slight subtlety in that we don't have write $(x)$. However, in the proof of Lemma 7, it suffices to use $n$ binary locations in each layer that are initialized to 0 , instead of 2 registers. The idea is that a process sets the $i$ 'th location to 1 to indicate that it had value $i-1$.
THEOREM 26. It is possible to solve $n$-consensus using only $O(n \log n)$ memory locations supporting only read () , write(1) or test-and-set(), and write $(0)$ or reset () .

\section{CONCLUSIONS AND FUTURE WORK}

In this paper, we defined a hierarchy based on the space complexity of solving consensus. We used consensus because it is a well-studied, general problem that seems to capture a fundamental difficulty of multiprocessor synchronization. Moreover, consensus is universal: any sequentially defined object can be implemented in a wait-free way using only consensus objects and registers [Her91].

We did not address the issue of universality within our hierarchy. One history object can be used to implement any sequentially defined object. Consequently, it may make sense to consider defining a hierarchy on sets of instructions based on their ability to implement a history object, a compare-and-swap object, or a repeated consensus object shared by $n$ processes. However, the space complexity of solving $n$-consensus is the same as the space complexity of implementing these long-lived objects, for many of the instruction sets that we considered. (It appears that this may not be true for $\{$ read, increment $\}$.)

It may be that a truly accurate complexity-based hierarchy would have to take time complexity into consideration. However it is even unclear which definition of time to use. Exploring this may be an important future direction.

There are several other interesting open problems. To the best of our knowledge, all existing space lower bounds rely on a combination of covering and indistinguishability arguments. However, when the covering processes apply $\operatorname{swap}(x)$, as opposed to write $(x)$, they can observe differences between executions, so they can no longer be reused to maintain indistinguishability. This means that getting a tighter space lower bound for $\{\operatorname{swap}(x), \operatorname{read}()\}$ would most likely require a completely novel approach. An algorithm that uses less than $n-2$ space would be even more surprising, as the processes would necessarily have to adapt their access patterns to the memory locations based on the swapped values, in order to circumvent the argument from [Zhu16]. The authors are unaware of any such algorithm.

We conjecture that, for sets of instructions, $\mathcal{I}$, which contain only read (), write $(x)$, and either fetch-and-increment () or increment ()$, \mathcal{S P}(\mathcal{I}, n) \in \Theta(\log n)$. We have a similar conjecture for $\mathcal{I}=\{\operatorname{read}()$, write $(0)$, write $(1)\}$ : we believe that $\mathcal{S P}(\mathcal{I}, n) \in \Theta(n \log n)$. Proving this is likely to require techniques that depend on the number of values, such as in the lower bound for $m$-valued adopt-commit objects in [AE14].

We would like to understand the properties of sets of instructions at certain levels in the hierarchy. For instance, what properties enable a collection of instructions to solve $n$-consensus using a single location? Is there an interesting characterization of the sets of instructions $\mathcal{I}$ for which $\mathcal{S P}(\mathcal{I}, n)$ is constant?

How do subsets of a set of instructions relate to one another in terms of their locations in the hierarchy? Alternatively, what combinations of sets of instructions decrease the amount of space needed to solve consensus? For example, using only read () , write $(x)$, and either increment () or decrement () , more than one memory location is needed to solve binary consensus. But with both increment () and decrement() a single location suffices. Are there general properties governing these relations? 


\section{ACKNOWLEDGMENTS}

Support is gratefully acknowledged from the Natural Science and Engineering Research Council of Canada, the National Science Foundation under grants CCF-1217921, CCF1301926, and IIS-1447786, the Department of Energy under grant ER26116/DE-SC0008923, and Oracle and Intel corporations.

The authors would like to thank Michael Coulombe, Dan Alistarh, Yehuda Afek, Eli Gafni and Philipp Woelfel for helpful conversations and feedback.

\section{REFERENCES}

[AAC09] James Aspnes, Hagit Attiya, and Keren Censor. Max registers, counters, and monotone circuits. In Proc. 28th ACM Symposium on Principles of Distributed Computing, pages 36-45, 2009.

$\left[\mathrm{AAD}^{+}\right.$93] Yehuda Afek, Hagit Attiya, Danny Dolev, Eli Gafni, Michael Merritt, and Nir Shavit. Atomic snapshots of shared memory. Journal of the ACM, 40(4):873-890, 1993.

[AE14] James Aspnes and Faith Ellen. Tight bounds for adopt-commit objects. Theory Comput. Syst., 55(3):451-474, 2014.

[AH90] James Aspnes and Maurice Herlihy. Fast randomized consensus using shared memory. Journal of Algorithms, 11(3):441-461, 1990.

[Bow11] Jack R. Bowman. Obstruction-free snapshot, obstruction-free consensus, and fetch-and-add modulo k. Technical Report TR2011-681, Computer Science Department, Dartmouth College, 2011.

http://www.cs.dartmouth.edu/reports/TR2011681.pdf.

[BRS15] Zohir Bouzid, Michel Raynal, and Pierre Sutra. Brief announcement: Anonymous obstruction-free $(n, k)$-set agreement with $n-k+1$ atomic read/write registers. In Proc. 29th International Symposium on Distributed Computing (DISC), page 669, 2015.

[FHS98] Faith Ellen Fich, Maurice Herlihy, and Nir Shavit. On the space complexity of randomized synchronization. Journal of the ACM, 45(5):843-862, 1998.

[FLMS05] Faith Ellen Fich, Victor Luchangco, Mark Moir, and Nir Shavit. Obstruction-free algorithms can be practically wait-free. In Proc. 19th International Conference on Distributed Computing (DISC), pages 78-92, 2005.

[Gel15] Rati Gelashvili. On the optimal space complexity of consensus for anonymous processes. In Proc. 29th International Symposium on Distributed Computing (DISC), pages 452-466, 2015.
[GHHW13] George Giakkoupis, Maryam Helmi, Lisa Higham, and Philipp Woelfel. An $\mathcal{O}(\sqrt{n})$ space bound for obstruction-free leader election. In Proc. 27th International Symposium on Distributed Computing (DISC), pages 46-60, 2013.

[GR05] Rachid Guerraoui and Eric Ruppert. What can be implemented anonymously? In Proc. 19th International Symposium on Distributed Computing (DISC), pages 244-259, 2005.

[Her91] Maurice Herlihy. Wait-free synchronization. ACM Transactions on Programming Languages and Systems (TOPLAS), 13(1):124-149, 1991.

[HR00] Maurice Herlihy and Eric Ruppert. On the existence of booster types. In Proc. 41st IEEE Symposium on Foundations of Computer Science (FOCS), pages 653-663, 2000.

[HS12] Maurice Herlihy and Nir Shavit. The Art of Multiprocessor Programming. Morgan Kaufmann, 2012.

[Int12] Intel. Transactional Synchronization in Haswell, 2012. http://software.intel.com/enus/blogs/2012/02/07/transactionalsynchronization-in-haswell.

[Jay93] Prasad Jayanti. On the robustness of herlihy's hierarchy. In Proc. 12th ACM Symposium on Principles of Distributed Computing (PODC), pages 145-157, 1993.

[LH00] Wai-Kau Lo and Vassos Hadzilacos. All of us are smarter than any of us: Nondeterministic wait-free hierarchies are not robust. SIAM Journal on Computing, 30(3):689-728, 2000.

[PR01] Rasmus Pagh and Flemming Friche Rodler. Cuckoo hashing. In Proc. 9th Annual European Symposium on Algorithms (ESA), pages 121-133, 2001.

[Ray12] Michel Raynal. Concurrent programming: algorithms, principles, and foundations. Springer Science \& Business Media, 2012.

[Rup00] Eric Ruppert. Determining consensus numbers. SIAM Journal on Computing, 30(4):1156-1168, 2000.

[Sch97] Eric Schenk. The consensus hierarchy is not robust. In Proc. 16th ACM Symposium on Principles of Distributed Computing (PODC), page 279, 1997.

[Tau06] Gadi Taubenfeld. Synchronization algorithms and concurrent programming. Pearson Education, 2006.

[Zhu15] Leqi Zhu. Brief announcement: Tight space bounds for memoryless anonymous consensus. In Proc. 29th International Symposium on Distributed Computing (DISC), page 665, 2015.

[Zhu16] Leqi Zhu. A tight space bound for consensus. In Proc. 48th ACM Symposium on Theory of Computing (STOC), 2016. To appear. 